\title{
Nuclear iASPP determines cell fate by selectively inhibiting either p53 or NF-KB
}

\author{
Wenjie Ge ${ }^{1,4}$, Yudong Wang ${ }^{1,4}$, Shanliang Zheng ${ }^{1}$, Dong Zhao ${ }^{1}$, Xingwen Wang ${ }^{1}$, Xiaoshi Zhang ${ }^{2 凶}$ and Ying Hu ${ }^{1,3}{ }^{凶}$ \\ (c) The Author(s) 2021
}

p53 and NF-кBp65 are essential transcription factors (TFs) in the cellular response to stress. Two signaling systems can often be entwined together and generally produce opposing biological outcomes in a cell context-dependent manner. Inhibitor of apoptosis-stimulating protein of p53 (iASPP) has the potential to inhibit both p53 and NF-KBp65, yet how such activities of iASPP are integrated with cancer remains unknown. Here, we utilized different cell models with diverse p53/NF-kBp65 activities. An iASPP (295-828) mutant, which is exclusively located in the nucleus and has been shown to be essential for its inhibitory effects on p53/ NF-KBp65, was used to investigate the functional interaction between iASPP and the two TFs. The results showed that iASPP inhibits apoptosis under conditions when p53 is activated, while it can also elicit a proapoptotic effect when NF-kBp65 alone is activated. Furthermore, we demonstrated that iASPP inhibited the transcriptional activity of p53/NF-kBp65, but with a preference toward p53, thereby producing an antiapoptotic outcome when both TFs were simultaneously activated. This may be due to stronger binding between p53 and iASPP than NF-KBp65 and iASPP. Overall, these findings provide important insights into how the activities of p53 and NF-KBp65 are modulated by iASPP. Despite being a well-known oncogene, iASPP may have a proapoptotic role, which will guide the development of iASPP-targeted therapies to reach optimal outcomes in the future.

Cell Death Discovery (2021)7:195; https://doi.org/10.1038/s41420-021-00582-1

\section{INTRODUCTION}

Mammalian cells are unavoidably and continually affronted by various stresses. To maintain tissue homeostasis, a diverse range of mechanisms have been developed. The stress response contributes to cellular decision-making processes governing cell death and is thus essential in human diseases, including cancer.

p53 and NF-KBp65 are key regulators of stress signaling. p53 plays a critical role in tumor suppression. Genomic mutations of p53 exist in about $50 \%$ of human cancers [1]. In contrast, NFKBp65 is often constitutively activated in cancers, highlighting the oncogenic identity of this gene [2]. Both proteins normally remain inactive under basal and unstressed conditions. Upon a variety of stresses, such as DNA damage [3], hypoxia [4], and oncogene activation [5], p53 is intensively modified. Some of the modifications can disrupt its interaction with E3 ligase MDM2 (murine double minute 2e), the principal cellular antagonist of p53, and protect p53 from proteasome-mediated degradation $[3,5,6]$. Stabilized p53 is translocated to the nucleus, where it transactivates target gene expression, leading to cell death, senescence, or metabolic reprograming dependent on the stress type, duration, and strength [7-9]. Cells with defective p53 not only promote carcinogenesis but also confer resistance to chemotherapeutic drug-induced cell death [10]. Stimuli such as viruses, cytokines, DNA damage, or antigen receptors can induce the activation of NF-KBp65 [11]. This is caused predominately by the phosphorylation and subsequent degradation of its cytosolic binding partner and inhibitor IKB (Inhibitor of kappaB) [12]. Once free of IKB binding, NF-KB translocates to the nucleus and functions as a transcription factor (TF) to promote the expression of genes that promote survival, proliferation, or inflammation, in turn favoring tumor development and chemoresistance [13-15].

Given the immense importance that the p53 and NF-KB pathways have in cellular physiology and pathology, it is not surprising that cross talk between these two transcriptional regulatory networks has been intensively studied. It is believed that the activation of NF-KB generally inhibits p53's function and vice versa $[16,17]$. However, NF-kBp65 has also been suggested to be required for p53-dependent apoptosis [18] and NF-KBp65 has been shown to activate p53 in inflammatory responses. Clearly, signaling mediated by $\mathrm{p} 53$ and NF-KB is often intertwined, leading to synergistic or antagonistic effects in a cellular context- and stimuli-dependent manner. Thus, unveiling how p53 and NF-kB are coordinately regulated is critically important, because it may help to identify and develop effective strategies to modulate cell fate, particularly in the context of tailored anticancer therapies.

A number of studies have provided evidence of selective activation of $\mathrm{p} 53$ or NF-kBp65. For example, the activation of Akt phosphorylates MDM2, leading to nuclear translocation of MDM2 and subsequent degradation of p53 in the nucleus [19]. Akt can also phosphorylate IKKB (inhibitor of nuclear factor-kB kinase

\footnotetext{
${ }^{1}$ School of Life Science and Technology, Harbin Institute of Technology, Harbin, Heilongjiang Province 150001, China. ${ }^{2}$ Department of Clinical Laboratory, Qilu Hospital of Shandong University, Jinan, Shandong 250012, China. ${ }^{3}$ Shenzhen Graduate School of Harbin Institute of Technology, Shenzhen 518055 , China. ${ }^{4}$ These authors contributed equally: Wenjie Ge, Yudong Wang. ${ }^{\infty}$ email: histone@126.com; huying@hit.edu.cn
} Edited by Ivano Amelio

Received: 19 May 2021 Accepted: 5 June 2021

Published online: 26 July 2021 


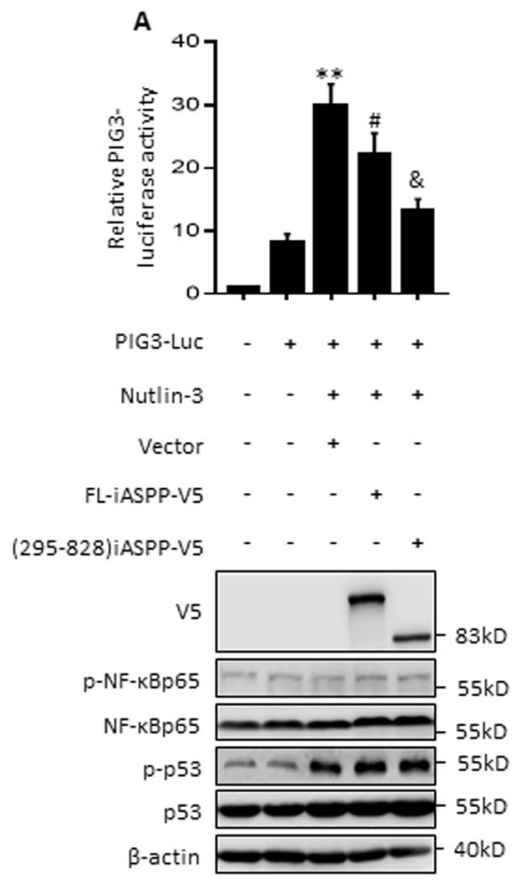

B
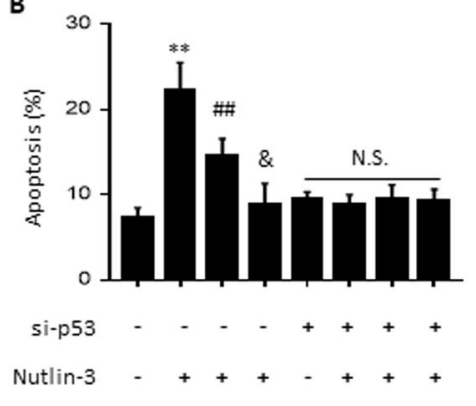

Vector - + - - + - -

FL-iASPP-V5 - $-+\cdots+-$

(295-828)iASPP-V5 - $-\quad+\quad-\quad+$

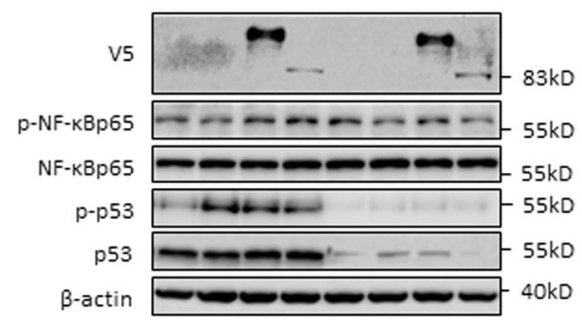

Fig. 1 iASPP fragment inhibits apoptosis in MCF-7 when p53 was activated by Nutlin-3. PIG3 luciferase activity (A) and apoptosis levels (B) were determined by the luciferase reporter assay and Annexin V-FITC and PI staining in MCF-7 cells after the indicated treatments. The PIG3 luciferase activity in cells transfected with the same amount of empty vector plasmid was normalized to 1 . Relative protein levels were determined by western blot. $\beta$-actin was used as an immunoblots loading control. ${ }^{* *} p<0.01$, relative to the untreated control; $\# p<0.05$ relative to the Nutlin-3-treated control; $\& p<0.05$ relative to Nutlin-3-treated FL control. N.S. not significant.

subunit beta), which activates signals leading to the degradation of IKB and the activation of NF-KBp65 [20]. In contrast, the tumor suppressor ARF (ADP-ribosylation factor) binds with MDM2 and disrupts its inhibitory effect on p53 [5], on the one hand; while promoting CHK1(checkpoint kinase1)-mediated NF-KBp65 phosphorylation, inducing NF-KBp65 nuclear translocation and activation [21], on the other. In addition, both p53 and NF-kBp65 have been shown to be acetylated by $\mathrm{p} 300 / \mathrm{CBP}$ (CREB binding protein) $[22,23]$. Such modification is essential for the activity of both TFs. It has been shown that the pool of p300/CBP in the nucleus is limited [24] and that the activities of p53 and NF-KBp65 can be determined by their competitive binding with p300/CBP [25]. IKB represents another direct contact between p53 and NF-KBp65 that can bind and recruit both factors in the cytoplasm, thus inhibiting their transcriptional activities in the nucleus [26]. Given the critical roles of the functional interaction between p53 and NF-KBp65, cells may use other points of contact to regulate the reciprocal relationship of $\mathrm{p} 53$ and NF-KBp65.

Of note, an inhibitor of apoptosis-stimulating protein of p53 (iASPP), an oncogene that was initially identified as an NF-KBp65binding partner and transcriptional inhibitor, has also been documented to bind with p53 and inhibit p53-dependent apoptosis in the nucleus. However, it remains unclear whether iASPP contributes to the cross talk between NF-KBp65 and p53 signaling $[27,28]$. Recently, we demonstrated that iASPP can be catalyzed by caspase- 3 after triggering apoptosis. The resultant iASPP(295-828) fragment is stable and translocates from the cytoplasm to the nucleus, where it exhibits better binding toward both p53 and NF-KBp65 and also has a more dramatic inhibitory effect on their transcriptional activities than full-length (FL) iASPP [29]. Nonetheless, it remains unclear how iASPP integrates its inhibitory effects on p53 and NF-KBp65, which generally lead to opposing biological outcomes. Here, we report that iASPP(295-828) simultaneously inhibits p53 and NF-KBp65 activity, thus enhancing NF-KBp65-regulated apoptosis but blocking p53-dependent apoptosis. We also describe that iASPP (295-828) has an intrinsic binding preference for $p 53$, suggesting that it may contribute to the selective activation observed between p53 and NF-KBp65 under stress.

\section{RESULTS}

iASPP(295-828) acts as an apoptotic inhibitor by blocking the transcriptional activity of p53

To understand the biological outcomes of nuclear iASPP activity in regulating $\mathrm{p} 53$ and NF-KBp65, we first examined the functional interaction between iASPP and p53 under conditions when p53 was activated but NF-KBp65 was inactivated. As shown, p53 was activated (indicated by increased p53 levels and PIG3 (p53 inducible protein 3) luciferase activity) after treatment of p53wild type MCF-7 cells with the MDM2 inhibitor Nutlin-3; however, treatment had no obvious effect on the expression levels of NFKBp65 or NF-kBp65 activation, as indicated by p-NF-KBp65 (Fig. 1A). Both FL-iASPP and iASPP(295-828) inhibited PIG3 luciferase activity, although the effect of $\operatorname{iASPP}(295-828)$ was more pronounced than that of FL-iASPP, with FL-iASPP reducing activity to $75 \%$ of the control and iASPP(295-828) to $40 \%$ (Fig. 1A).

We next used Annexin $\mathrm{V}$ and propidium iodide (PI) double staining to analyze apoptotic percentages under the same conditions. Nutlin-3 induced apoptosis by about 2.8 -fold, while iASPP(295-828) reduced the effect of Nutlin-3 by about $60 \%$ ( $p<$ 0.05 , Fig. 1B). Small interfering RNA (siRNA) specifically targeting p53 inhibited p53 expression, as confirmed by western blot. Accordingly, it completely abrogated Nutlin-3-induced apoptosis, as expected. Remarkably, both FL-iASPP and iASPP(295-828) lost their abilities to inhibit apoptosis after p53 knockdown (Fig. 1B). Consistently, we found that 5-FU induced mild levels of apoptosis in HEK293 cells and that p53 overexpression increased 5-FUinduced apoptosis levels. Additional ectopic expression of FLiASPP or iASPP(295-828) inhibited p53-elevated apoptosis, and 
A

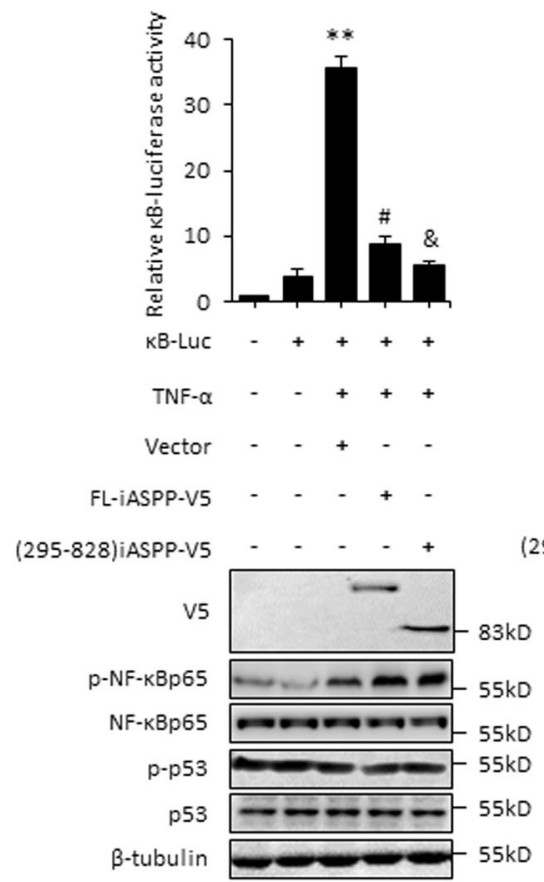

B

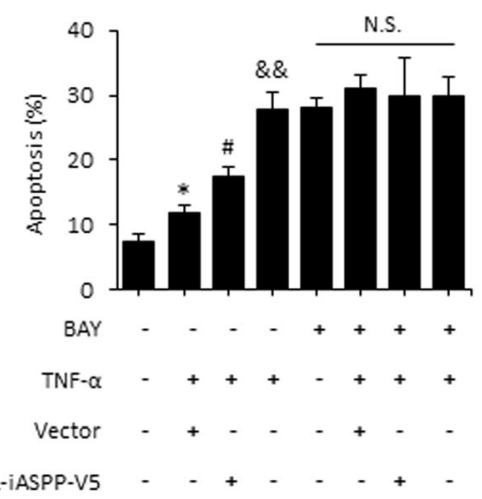

295-828)iASPP-V5 - $-\quad+\quad-\quad+$

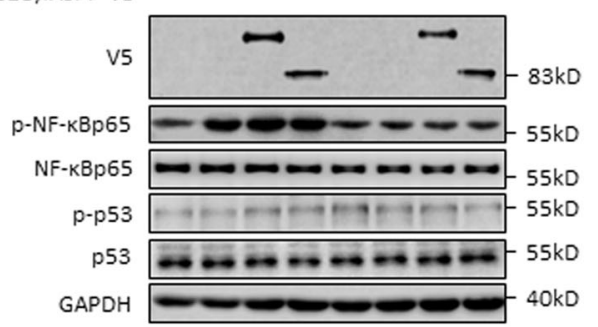

Fig. 2 iASPP fragment promotes apoptosis in MCF-7 when NF-KBp65 was activated by TNF-a. MCF-7 cells were transfected as indicated and treated with or without $10 \mathrm{nM}$ TNF- $\alpha$ in the presence or absence of $10 \mu \mathrm{M}$ BAY117082 (BAY), then the transcriptional activity of NF- $\kappa$ Bp65, as indicated by $\kappa B$ luciferase activity $(\mathbf{A})$ or apoptosis levels $(\mathbf{B})$, were measured. The $\kappa B$ luciferase activity in cells transfected with the same amount of empty vector plasmid was normalized to 1 . Relative protein levels were determined by western blot. $\beta$-tubulin was used as an immunoblots loading control. ${ }^{*} p<0.05,{ }^{* *} p<0.01$, relative to the untreated control; $\# p<0.05$ relative to the TNF- $\alpha$-treated control; \& $p<0.05$, $\& \& p<0.01$ relative to TNF- $\alpha$-treated FL control. N.S. not significant.

the effect was more evident with iASPP(295-828) than FL-iASPP (Fig. S1). These data suggest that iASPP(295-828) can inhibit apoptosis more efficiently than FL-iASPP by inhibiting p53's transcriptional activity under conditions when p53 is activated and NF-kBp65 remains inactive.

\section{iASPP(295-828) has the ability to promote apoptosis by inhibiting the transcriptional activity of NF-kBp65}

iASPP was first identified as being able to bind to and inhibit an NF-kBp65 subunit; however, the biological significance of this interaction in the context of apoptotic regulation is not yet known. Given the observed increased inhibition caused by iASPP (295-828), we further investigated the impact of iASPP(295-828) on apoptosis in the context of NF-KBp65 activation. To this end, TNF- $a$ (tumor necrosis factor- $\alpha$ ) was applied to activate NF-kBp65 in MCF-7 cells [30]. The results showed that TNF- $a$ activated NFKBp65, as indicated by KB-luciferase activity and p-NFKBp65 levels, which had no obvious impact on p53 (Fig. 2A). iASPP and iASPP (295-828) reduced $\mathrm{kB}$-luciferase activity by about $85 \%$, which was more efficient than the effect of FL-iASPP (Fig. 2A). Also notably, FL-iASPP tans-localized into the nucleus after TNF-a treatment (Fig. S2A), which suggests that nuclear localization may be required for iASPP to inhibit NF-KB. TNF-a produced no obvious effect on cell proliferation, as indicated by BrdU incorporation rate (Fig. S2B). Inhibiting NF-KB activity by BAY117082 (BAY) failed to influences cell proliferation either (Fig. S2B). However, TNF-a induced mild levels of apoptosis. Interestingly, ectopic FL-iASPP expression promoted apoptosis by about 2.1-fold and similar levels of ectopically expressed iASPP(295-828) elicited a more dramatic proapoptotic effect in the presence of TNF-a $(p<0.01)$ (Fig. 2B).

To further investigate whether the proapoptotic effects of iASPP and its mutant were due to their ability to inhibit NF-KBp65, we examined the effects of iASPP in the presence of the NF-KBp65 inhibitor BAY. As shown, the p-NF-kBp65 protein was repressed by $B A Y$, and BAY completely blocked the proapoptotic effect of iASPP in TNF-a-treated cells (Fig. 2B). In addition, in contrast to p53, NFкBp65 overexpression abrogated 5-FU-induced apoptosis in HEK293 cells. Overexpression of FL-iASPP had no obvious impact on NF-kBp65-inhibited apoptosis, while iASPP(295-828) completely abrogated the effect of NF-kBp65 (Fig. S3A). These data suggest that iASPP(295-828) also exhibits proapoptotic activity by inhibiting the activity of NF-KBp65 under conditions when NFKBp65 is activated.

To further validate the findings described above, the regulatory effects of iASPP(295-828) on apoptosis were also examined in the breast cancer cell line SK-BR3, in which NF-KBp65 has been reported to be constitutively active and p53 has been inactivated by mutation [31, 32]. Interestingly, iASPP(295-828) alone significantly reduced the cellular viability of SK-BR3 cells in a timedependent manner after transfection (Fig. $3 \mathrm{~A}$ ). This was mainly due to increased apoptosis because the proportions of Annexin V/ $\mathrm{PI}$-positive cells were markedly increased (Fig. 3B). Proapoptotic Bax (Bcl-2 associated $\mathrm{X}$ ) was elevated and antiapoptotic Bcl-2 (Bcell lymphoma-2) was repressed (Fig. 3C). However, the cell cycle distribution was not disrupted under the above conditions (Fig. 3D). Importantly, this effect of iASPP(295-828) on cell viability and apoptosis was found to be completely abolished by either BAY (Fig. 4A, B) or siRNA-mediated NF-kBp65 knockdown (Fig. 4C). By contrast, FL-iASPP had no obvious impact on cellular viability. Subcellular fractionation assay revealed that FL-iASPP was predominately localized in the cytoplasm under basal conditions (Fig. S3B), suggesting that nuclear localization may be required for its activity to inhibit NF-kBp65.

Moreover, SK-BR3 cells are well known to be highly resistant to chemotherapeutic agents such as paclitaxel [33]. Constitutively 


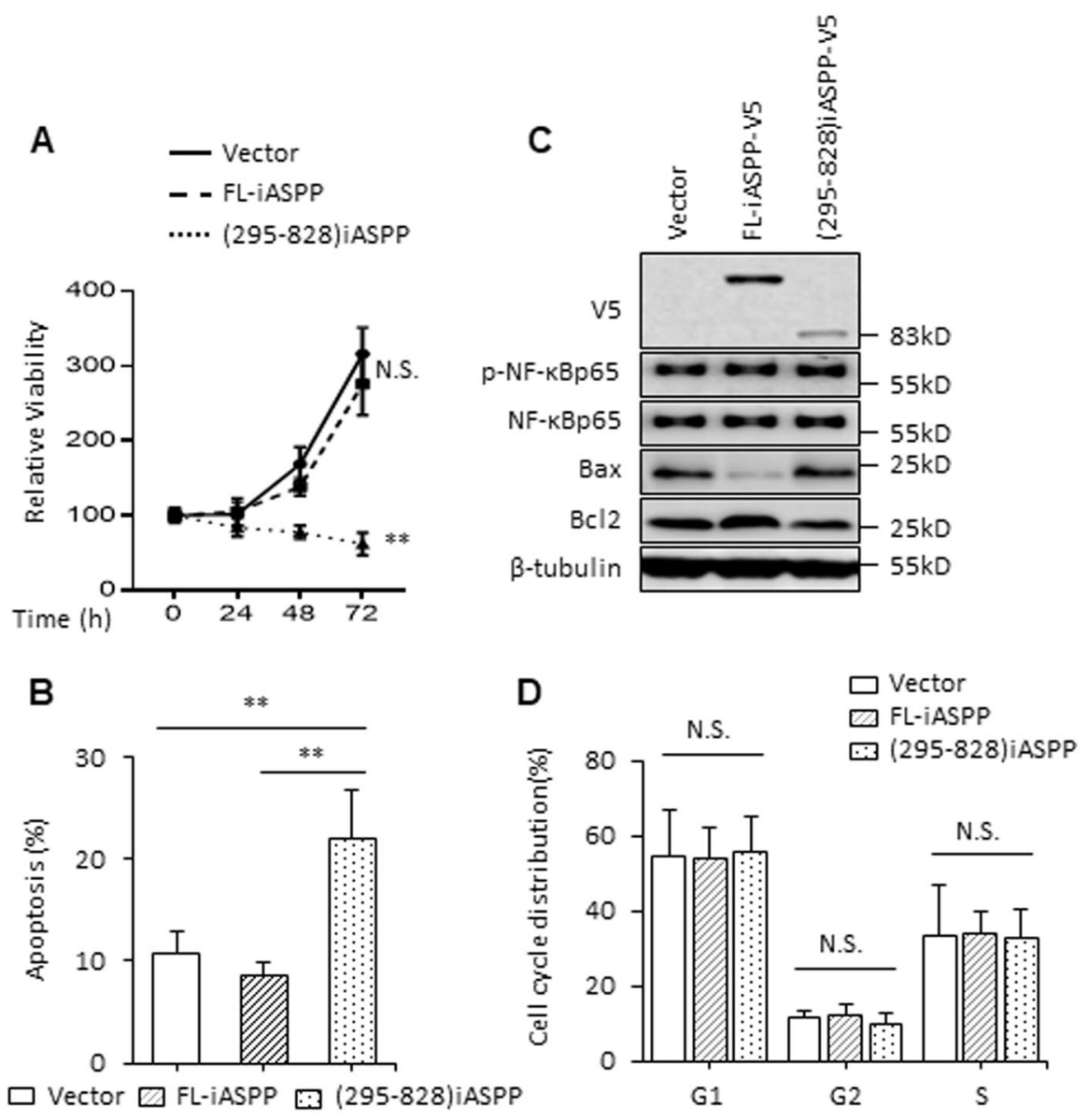

Fig. 3 The cleaved iASPP fragment promotes apoptosis in NF-KBp65 constitutively activated SK-BR3. A SK-BR3 cells were transfected with Vector, FL-iASPP, and (295-828) iASPP plasmids, then cell viability was revealed by MTT assay. ${ }^{* *} p<0.01$, N.S. not significant. B Apoptosis levels were determined by Annexin V-FITC and PI staining in SK-BR3 cells after the indicated treatments. ${ }^{* *} p<0.01$. C Bax, Bcl-2, p-NF-kBp65, and NFкBp65 were determined by western blot in SK-BR3 cells. $\beta$-tubulin was used as an immunoblots loading control. D Cell cycle arrest was revealed by PI staining in SK-BR3 cells after the indicated treatments. N.S. not significant.

active NF-кBp65 may contribute to the resistance phenotype [34]. Given the fact that iASPP(295-828) is able to inhibit NF-кBp65, we speculated that iASPP(295-828) may sensitize cells to paclitaxelinduced apoptosis. Indeed, iASPP(295-828) markedly reduced cell viability after paclitaxel treatment (Fig. 5A). BAY or si- NF-kBp65 also increased paclitaxel-induced apoptosis, while no further effect was detected with a combination of iASPP(295-828) overexpression and BAY/ si- NF-KBp65 (Fig. 5B, C), suggesting that iASPP(295-828) sensitizes cells to paclitaxel-induced apoptosis by inhibiting the activity of NF-kBp65.

Taken together, the nuclear iASPP mutant iASPP(295-828) has the potential to promote apoptosis by inhibiting NF-KBp65 under conditions where NF-KBp65 is active and p53 is inactive.

\section{iASPP(295-828) preferentially inhibits p53 over NF-KBp65}

Based on the fact that iASPP(295-828) acts as a double-edged sword in regulating apoptosis, by inhibiting either p53 or NFKBp65, we next investigated whether iASPP(295-828) preferentially regulates either of these two targets. For this purpose, we utilized a model where both TFs were simultaneously activated by treating RKO cells with actinomycin D (ActD) [18]. As shown, p53 and NF-KBp65 were both activated, as indicated by the luciferase reporter assay or elevated p-NF-KBp65 and p-p53 expression (Fig. $6 \mathrm{~A}, \mathrm{~B})$. Interestingly, when the transcriptional activities of p53 and NF-kBp65 were elevated by ActD to similar extents (twofold), iASPP(295-828) reduced $\mathrm{KB}$-luciferase activity to about $83 \%$ of the control, while PIG3 luciferase activity was reduced to $\sim 19 \%$ of the control ( $p<0.01$, Fig. $6 \mathrm{~A})$. The preferential p53 inhibitory activity of iASPP(295-828) was also observed in ActD-treated HCT116 cells, suggesting it is a cell-type-specific event (Fig. S4A, B). In keeping with the above data, iASPP(295-828) inhibited apoptosis by about $50 \%$ in ActD-treated RKO cells ( $p<0.05$, Fig. 6B).

A ChIP assay revealed that the binding of p53 with the promoter region of its proapoptotic target Bax was reduced after iASPP(295-828) overexpression by approximately threefold under unstressed conditions and by eightfold under ActD-treated conditions (Fig. 6C). In contrast, the binding of NF-KBp65 and its antiapoptotic target $\mathrm{Bcl}-2$ was reduced to a much lesser extent. These data were further validated by the mRNA expression of these targets as detected by real-time RT-PCR (Fig. 6D). These results indicate that iASPP(295-828) binds p53 more effectively than NF-KBp65 and thus has a more dramatic effect on p53.

\section{iASPP(295-828) binds with p53 more efficiently than with NF-} KBp65

It has been proposed before that preferential binding of p300 may contribute to the selective activation of p53 and NF-KBp65. We asked whether iASPP prefers to inhibit p53 than NF-kB by modulating p300; however, this is unlikely to be the case, because p300 was not detected in RKO cells (Fig. S5A), in which iASPP was found to inhibit p53 than NF-KBp65 more efficiently (Fig. 6C, D).

We and others previously showed that iASPP/iASPP(295-828) inhibits $\mathrm{p} 53$ or NF-KBp65 activity by direct protein-protein interaction. Given that iASPP inhibits p53 relatively efficiently, 


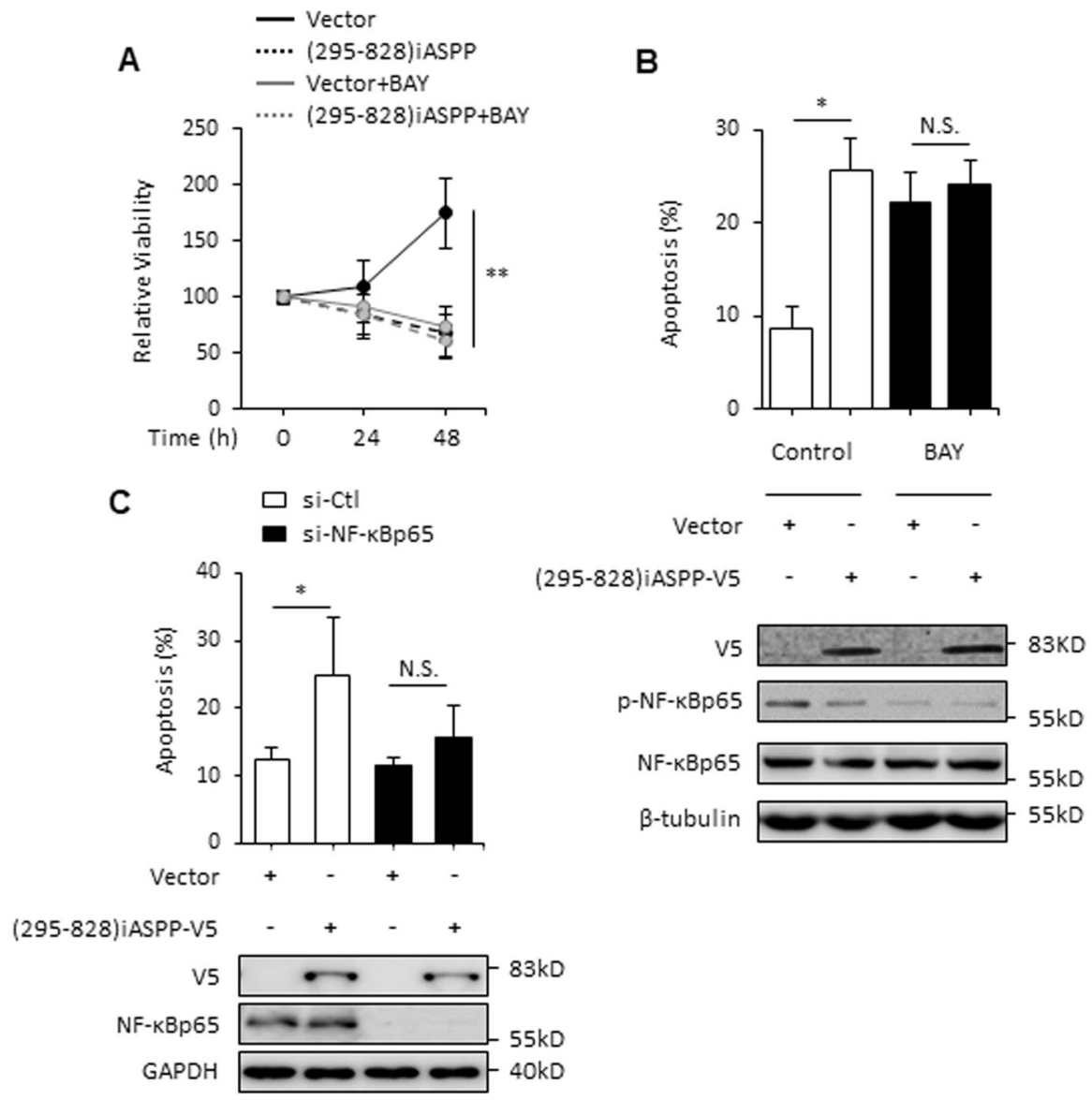

Fig. 4 iASPP fragment promotes apoptosis through NF-KBp65 in SK-BR3. A SK-BR3 cells were transfected as indicated and treated with or without $10 \mu \mathrm{M}$ BAY, then cell viability was revealed by MTT assay at $0,24,48 \mathrm{~h}$. ${ }^{* *} p<0.01$. B, C Apoptosis was determined by Annexin V-FITC and PI staining and immunoblots in SK-BR3 cells after the indicated treatments. GAPDH and $\beta$-tubulin were used as immunoblot loading controls. ${ }^{*} p<0.05$, N.S. not significant.

we reasoned that iASPP may bind with p53 and NF-KBp65 differently. To test this hypothesis, an in vitro immunoprecipitation assay was conducted. Plasmid constructs expressing either p53 or NF-KBp65 were generated, both with a myc tag. The in vitrosynthesized $\mathrm{p} 53$ and NF-KBp65 proteins were monitored by western blotting using an anti-myc antibody. Similar levels of p53 and NF-KBp65 proteins were mixed with the same amount of in vitro-translated iASPP-v5 protein. iASPP was precipitated with an anti-v5 antibody. Co-precipitated p53 or NF-kBp65 were detected using an anti-myc antibody. The results showed that coprecipitated p53-myc was much more prevalent than NF-kBp65myc (eightfold, $p<0.01$, Fig. 7A, B). Therefore, we concluded that iASPP binds with p53 better than with NF-kBp65.

Recently, we reported that FL-iASPP translocates to the nucleus during cellular senescence; meanwhile, p53 and NF-KBp65 were simultaneously activated [35]. Under such conditions, we noted that iASPP's ability to inhibit p21-luciferase reporter activity (a well-known p53 target) more efficiently than that of kB-luciferase reporter activity (Fig. S5B-D). In addition, the levels of an $80 \mathrm{kDa}$ iASPP were increased in colon cancer tissues compared with the normal controls (Fig. S5E). These data are in line with our data shown above.

\section{DISCUSSION}

p53 and NF-kBp65 are central to the cell's response to a variety of stresses, are linked to multiple fundamental biological processes, and thus play essential roles in carcinogenesis and drug resistance
[11, 36-38]. Complicated cross talk between p53 and NF-kBp65 mediates the balance between life and death, but relatively few studies have explored the molecular mechanisms involved. It would be logical to propose that key elements in their regulatory networks may contribute to the selective activation of p53 and NFKBp65, such as p300/CBP and IKB $[25,26]$. Here, we tested this idea and revealed that a previously identified p53 and NF-KBp65 inhibitor, iASPP, can produce an anti- or proapoptotic effect depending on which factor, p53 or NF-KBp65, is predominately activated in cells. In addition, iASPP favors binding with p53 and therefore has a more dramatic inhibitory effect on p53 than NFкBp65 when both are active.

We recently demonstrated that iASPP is mainly distributed in the cytoplasm under unstressed conditions in the majority of cancer types, where it elicits oncogenic activity by stabilizing antioxidative Nrf2 in a p53-independent manner [39]. It has also been reported that iASPP can produce an inhibitory effect on p53 in the nucleus because it loses its ability to bind with p53 in the cytoplasm due to iASPP dimer formation, which covers both iASPP's p53-binding motif and its nuclear translocation signal [40]. Thus, in order to better understand the functional interaction between p53 and iASPP, we have to define the conditions under which iASPP is retained in the nucleus. Of interest, we previously found that the activation of caspases facilitated the cleavage of iASPP during apoptosis. The stable iASPP(295-828) fragment is mainly distributed in the nucleus because of the loss of the $\mathrm{N}$ terminal residues that mediate iASPP's dimerization. Notably, in this nuclear iASPP(295-828) mutant, the sites that contribute to its 


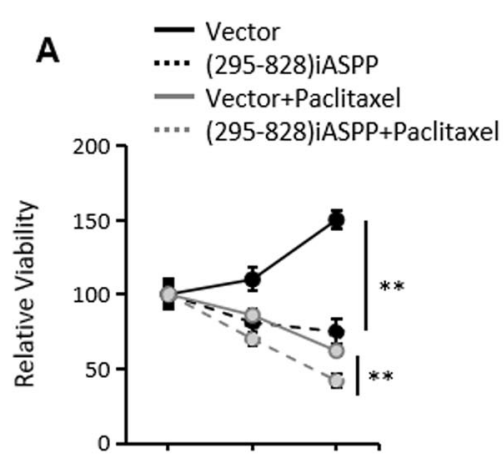

B

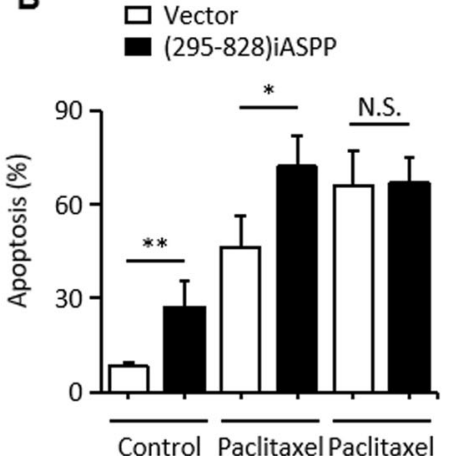

Control Paclitaxel Paclitaxel Control Paclitaxel
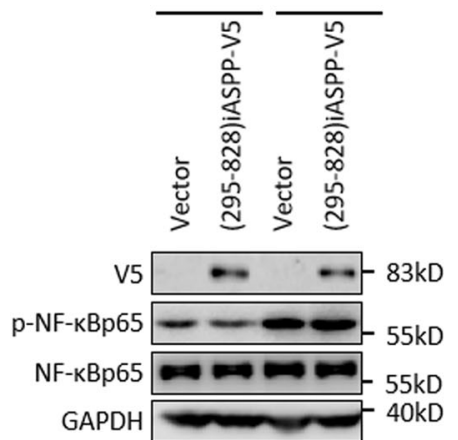
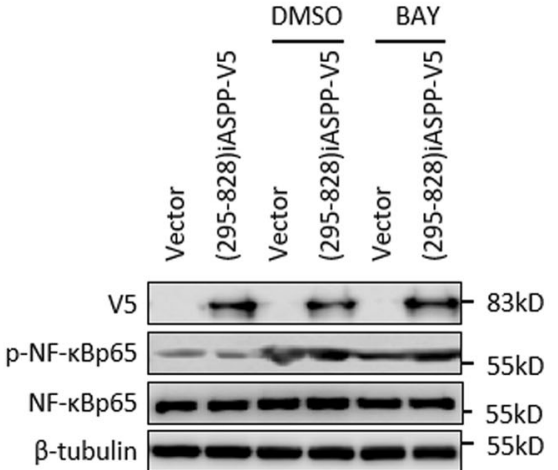

C

$\square$ Vector (295-828)iASPP

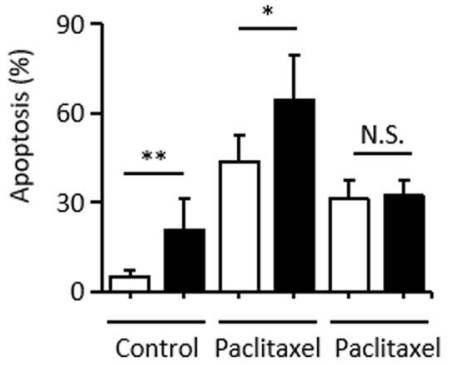

Control Paclitaxel Paclitaxel
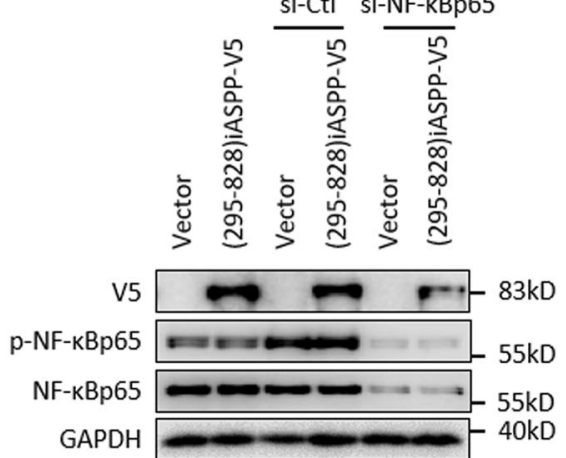

Fig. 5 NF-KBp65 is required for iASPP fragment enhanced paclitaxel sensitivity in SK-BR3. SK-BR3 cells were transfected as indicated and treated with or without $5 \mu \mathrm{M}$ paclitaxel, then cell viability $(\mathbf{A})$ or apoptosis levels (B, C) were revealed by MTT assay or Annexin V-FITC and PI staining. Relative protein levels were determined by western blot. GAPDH and $\beta$-tubulin were used as an immunoblots loading control. ${ }^{*} p<$ $0.05,{ }^{* *} p<0.01$, N.S. not significant.

binding with both $\mathrm{p} 53$ and NF-KBp65 are retained [29]. Indeed, our data revealed that iASPP(295-828) binds with both p53 and NF-KBp65 and inhibits their transcriptional activities. In addition, such activities of iASPP(295-828) were much stronger than those of FL-iASPP, supporting the idea that the nuclearly localized iASPP monomer is required for its inhibitory effect on p53. Nonetheless, the biological outcomes induced by iASPP(295-828) are not well understood. Findings from the present study show that iASPP, a well-established oncogene, is actually able to promote apoptosis through the inhibition of NF-kBp65 under certain conditions. In addition, apoptotic cancer cells contain iASPP(295-828)

NF-KBp65 can be activated by TNF- $a$ and chemotherapeutic drugs, which is generally associated with apoptotic tolerance [41]. However, inhibition of NF-kB activation by expression of a dominant-negative mutant form of IKBa $(\mathrm{IKBm})$, which is resistant to phosphorylation and degradation, is not always able to increase the sensitivity to cytotoxic treatment [26], suggesting that additional levels of regulation of NF-KBp65 also exist. iASPP has been shown to inhibit NF-KBp65 both in vivo and in vitro $[42,43]$; however, its activity remains unknown in the context of apoptosis. Here, our data clearly show that iASPP promotes TNF-a- and paclitaxel-induced apoptosis via inhibition of NF-KBp65. In agreement with the proapoptotic function identified by us, Kramer and colleagues have also reported that iASPP can promote apoptosis by stabilizing p300/CBP [44]. It is known that there are many "double-edged sword" genes in the human genome. Our data and those of others suggest that iASPP may be one such gene. Thus, caution needs to be taken in the development of iASPP-targeted therapies, because cell context needs are taken into consideration.

In addition, we found that iASPP's binding with p53 is better than its binding with NF-KBp65 in an in vitro assay, and thus facilitates an antiapoptotic outcome in response to activation of both p53 and NF-KB. p53 and NF-KB share similar binding sites on
iASPP (the SH3 domain and ankyrin repeats) [28], and it will be interesting to investigate whether p53 has a greater binding affinity with iASPP and thus competitively blocks its binding with NF-kBp65. This model needs to be validated in future studies. Nonetheless, the potential difference in its binding with p53 and NF-KBp65 opens up opportunities for the design of specific inhibitors to block iASPP's interaction with p53 and strengthen its interaction with NF-KBp65. Thus, modulation of iASPP may become a useful strategy for the effective combination of p53 activation and NF-KBp65 inhibition.

Furthermore, we have investigated nuclear iASPP activity via ectopic expression of an iASPP nuclear mutant and with particular stimuli. However, FL-iASPP has been found to localize in the nucleus under certain circumstances. For example, M. Lu et al. have shown that iASPP predominately resides in the nucleus in some melanoma cell lines and patient samples. This is mainly due to the constitutive activation of cyclinB1/CDK1(cyclin-dependent kinase 1), which phosphorylates iASPP at key residues that mediate iASPP dimer formation [40]. We have also found that low-dose sublethal DNA damaging agents can promote the nuclear translocation of iASPP [35]. Here, we provide a potential explanation for why iASPP mainly behaves as an oncogene under the above conditions. Our results also revealed that the nuclearlocalized FL-iASPP inhibits p53 more efficiently than NF-kBp65, suggesting that nuclearly localized FL-iASPP's behavior is similar to that of the nuclear mutant. In addition, $80 \mathrm{kDa}$ iASPP fragment is produced in the apoptotic malignant cells [29]. We also detected an endogenous $80 \mathrm{kDa}$ iASPP in human tissues by an anti-iASPP antibody. The identity and the biological functions of this iASPP fragment need to be explored in the future.

In summary, iASPP can act as either a pro- or antiapoptotic factor, depending on whether p53 or NF-KBp65 is activated in the nucleus. Nonetheless, its ability to bind to and inhibit p53 is much stronger than to NF-KBp65 in nature. This study provides 


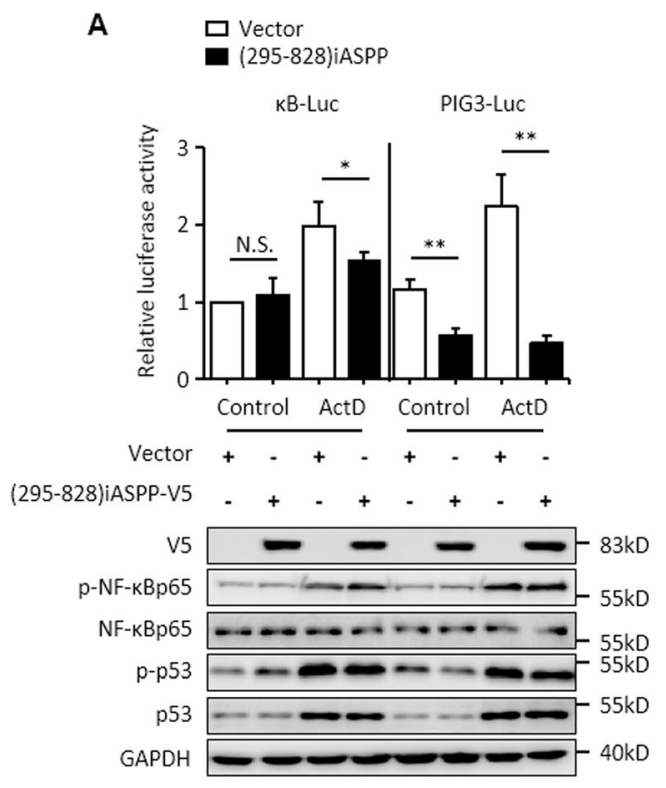

C

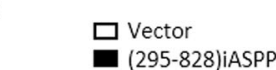

B

$\square$ Vector

(295-828)iASPP

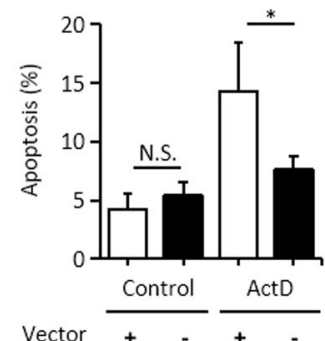

(295-828)iASPP-V5 - + - +

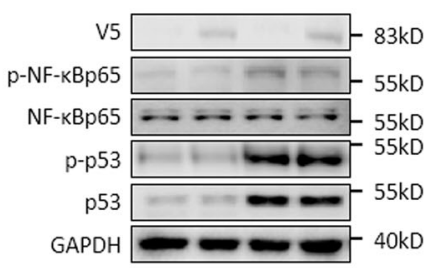

D

$\square$ Vector

(295-828)iASPP
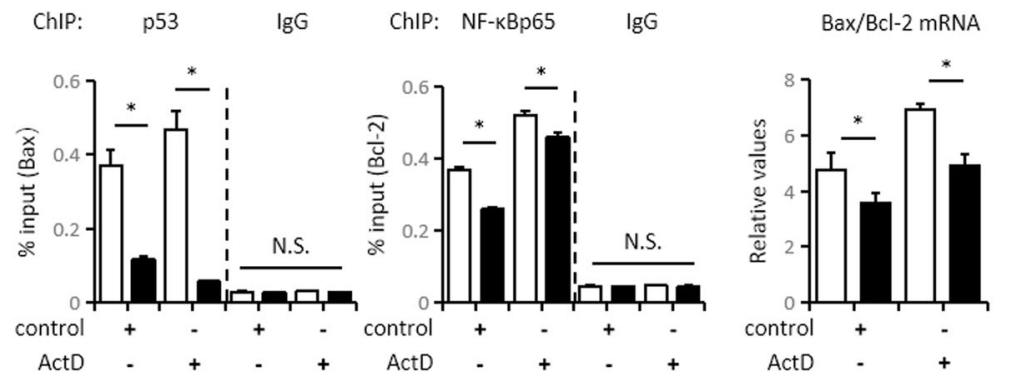

Fig. 6 iASPP(295-828) preferentially inhibits p53 over NF-kBp65. A, B NF-кBp65 and p53 transcriptional activities, as indicated by $\kappa B$ and PIG3 luciferase activity, were measured by a luciferase reporter assay (A) and apoptosis levels were determined by Annexin V-FITC and PI staining (B) in RKO cells after the indicated treatments. The transfection efficiency and p-p53 and p-NF-kBp65 activation were determined by immunoblots. GAPDH was used as an immunoblots loading control. ActD actinomycin D. ${ }^{*} p<0.05,{ }^{* *} p<0.01$, N.S. not significant. C, D RKO cells were transfected as indicated and treated with or without $7.5 \mathrm{nM} \mathrm{ActD}$, ChIP assay was used to detect the binding of p53 and NF-kBp65 with Bax and Bcl-2 promoter (C). The mRNA levels of Bax and Bcl-2 were determined by qRT-PCR. GAPDH was used as a loading control (D). ${ }^{*} p<0.05$, N.S. not significant.

important insights into the selective regulation of p53 and NF$\mathrm{KBp} 65$, and also provides guidance for the future development of iASPP-targeting therapies.

\section{MATERIALS AND METHODS}

\section{Cell culture}

RKO cells were maintained in RPMI-1640 medium (Gibco) supplemented with $10 \%(\mathrm{v} / \mathrm{v})$ fetal bovine serum (Biological Industries) and $2 \mathrm{mM} L-$ glutamine. MCF-7 and SK-BR3 cells were maintained in DMEM with the same supplement as shown above. All cell lines were grown at $37^{\circ} \mathrm{C}$ in the humidified incubator (Thermo Scientific) with $5 \% \mathrm{CO}_{2}$. Cell lines were routinely tested to exclude mycoplasma contamination and characterized by Genetic Testing Biotechnology Corporation (Suzhou, China) using short tandem repeat markers.

\section{Small interfering RNA (siRNA) and plasmid transfection}

siRNAs specifically targeting $\mathrm{p} 53, \mathrm{NF}-\mathrm{kBp} 65$, and nonspecific si-control were synthesized by GenePharma company. The sequences were shown as follows (si-p53: 5'-GAAGAAAATTTCCGCAAAA-3', si-NF-kBp65: 5'-CAUCAACUAUGAUGAGUUUCC- $3^{\prime}$ ). Full-length iASPP was kindly provided by Professor Xin Lu, Oxford University. Full-length NF-KBp65 and p53 were sub-cloned into a pCDNA3-myc3 vector using appropriate restriction enzyme digests. Plasmids expressing iASPP fragment (295-828) was generated by using Fast Mutagenesis System (Transgene biotech FM11102) with full-length iASPP expressing plasmid as a template. All transfection experiments were conducted by using Lipofectamine 2000 (Invitrogen) following the manufacturer's instructions.

\section{Western Blot (WB) assay}

Cells were lysed in UREA buffer (8 M Urea, $1 \mathrm{M}$ Thiourea, $0.5 \%$ CHAPS, $50 \mathrm{mM}$ DTT, and $24 \mathrm{mM}$ Spermine). Some amounts of protein lysates were subjected to SDS gel electrophoresis. The immune complex was detected by an ECL kit (Thermo Scientific). The v5 antibody was purchased from BioRad(Cat: MCA1360). p53(Cat:60283-2-lg), NF-kBp65(Cat:10745-1-AP), myc (Cat:16286-1-AP), and loading control antibodies, GAPDH(Cat:60004-1-lg), a-tubulin(Cat:66031-1-lg), $\beta$-tubulin(Cat:10094-1-AP), and $\beta$-actin(Cat:6600 9-1-lg) antibodies were from Proteintech Ltd. p-p53 (Ser15) and p-NFKBp65 (Ser536) antibody was brought from CST (Cat:12571, 3033). Bax and $\mathrm{Bcl}-2$ were from Abclonal (cat: A0207 and A0208, respectively). Anti-iASPP antibody (LX49.3) was brought from Merck (cat: A4605).

\section{In vitro translation and immunoprecipitation}

In vitro transcription and translation of plasmids were performed by using the Promega TNT T7 Quick coupled transcription/translation system (L1171, Promega) following the manufacturer's instructions. The in vitro 

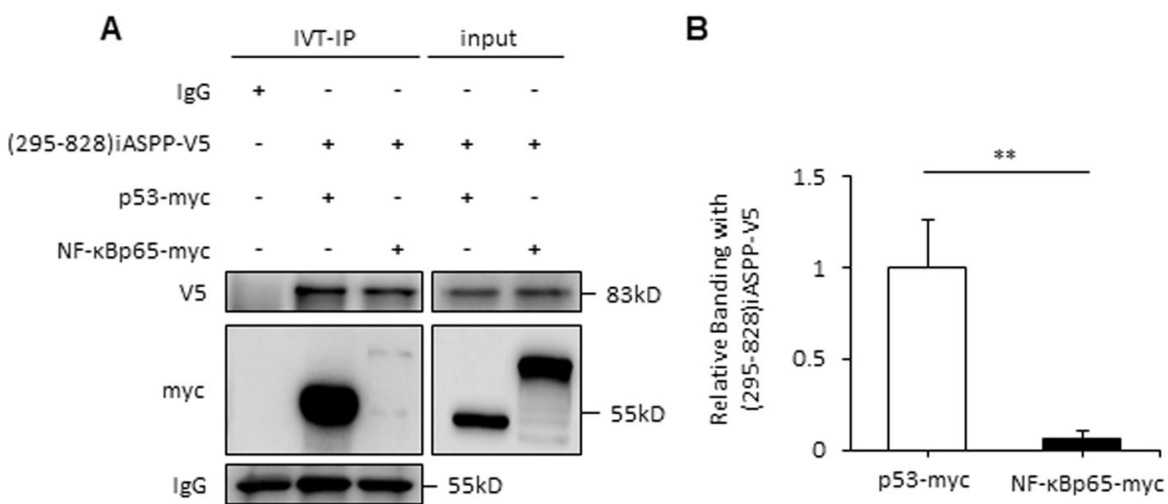

Fig. 7 iASPP(295-828) binds with p53 more efficiently than with NF-KBp65. The interaction between (295-828)iASPP and p53 and NF-

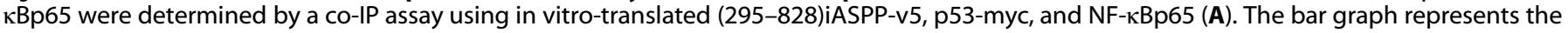
relative p53 or NF-kBp65 proteins that bound with (295-828)iASPP (B). ${ }^{* *} p<0.01$.

translated proteins were diluted in PBS. Immunoprecipitation was performed using ANTI-v5 (Bio-Rad). Equal amounts of lysate or IP complexes were resolved by $8 \%$ SDS-PAGE and then analyzed by WB assay.

\section{MTT}

Cell proliferation rate was assessed by MTT assay. Briefly, MTT solution in $1 \times$ PBS was added to each well at the final concentration of $0.5 \mathrm{mg} / \mathrm{ml}$. The plate was incubated for $3 \mathrm{~h}$ at $37^{\circ} \mathrm{C}$. The MTT medium was aspirated carefully and the dark-blue formazan was solubilized with DMSO. Optical density was measured with a spectrometer at $490 \mathrm{~nm}$. Each experiment was conducted in triplicates and repeated independently 3 times.

\section{Luciferase reporter assay}

Briefly, the same amounts PIG-3-, KB-, or p21-luciferase reporter together with either full length iASPP, iASPP(295-828) were transfected into the indicated cancer cells, respectively. Each of the transfections was included the same amount of Renilla, which was used to standardize transfection efficiency. Forty-eight hours after transfection, the luciferase activities in cell lysates were measured with the luciferase assay system (Promega). The relative luciferase reporter activity was normalized to the activity unit from the Renilla luciferase.

\section{Subcellular fractionation assay}

Cytoplasm lysis buffer (10 mM HEPES pH 7.9, $10 \mathrm{mM} \mathrm{KCl}, 1.5 \mathrm{mM} \mathrm{MgCl}$, and $0.5 \mathrm{mM}$ b-mercaptoethanol) was applied to cells, followed by moderate vortex for $15 \mathrm{~s}$ and $15-20$ min incubation on ice. Additional $5 \mu \mathrm{l} 10 \%$ NP-40 (Amersco) was then added to the mixture followed by another round of vortex and incubation. The cytoplasm fraction was obtained by collecting supernatant after centrifugation at $16,000 \mathrm{xg}$ for $10 \mathrm{~min}$ at $4{ }^{\circ} \mathrm{C}$. The resulting pellet was lysed in the nuclear fraction buffer (10 mM HEPES pH 7.6, 1 mM DTT, 7.5 mM MgCl2, 0.2 mM EDTA, $0.3 \mathrm{M} \mathrm{NaCl}$, $1 \mathrm{M}$ UREA, and $1 \% \mathrm{NP}-40)$. The supernatant was collected as the nuclear fraction by centrifugation at $16,000 \mathrm{xg}$ for $10 \mathrm{~min}$ at $4^{\circ} \mathrm{C}$.

\section{Apoptosis assay}

After indicated treatments, both suspension and attached cells were collected gently. Cell density was adjusted to $5 \times 10^{6}$ cells $/ \mathrm{ml}$. About $100 \mu \mathrm{l}$ cell suspension was incubated with $2.5 \mu \mathrm{l}$ AnnexinV/FITC for $10 \mathrm{~min}$ and then $2.5 \mu \mathrm{PI}$ (BD Pharmingen) for $5 \mathrm{~min}$ at room temperature in dark. The rate of apoptosis was measured by flow cytometry (BD Pharmingen).

\section{Chromatin immunoprecipitation (ChIP)}

Chromatin immunoprecipitation (ChIP) assay was performed to estimate protein binding at promoter regions of the target genes. Briefly, $1 \times 10^{7}$ cells were collected in lysis buffer $A$ and $B$ (buffer A: $5 \mathrm{mM}$ PIPES, $85 \mathrm{mM} \mathrm{KCl}$, and $0.5 \%$ NP40. buffer B: $1 \%$ SDS, $10 \mathrm{mM}$ EDTA, and $50 \mathrm{mM}$ Tris- $\mathrm{HCl}$.) supplemented with Protease Inhibitor Cocktail and sonicated to generate chromatin samples with average fragment sizes of $100-500 \mathrm{bp}$. Cell lysates were treated with the indicated antibodies or IgG control at $4{ }^{\circ} \mathrm{C}$ overnight. Then, the supernatants were mixed with the blocked Protein A/D sepharose beads to collect the antibody-chromatin complexes. After washing four times in wash buffers (low salt buffer, high salt buffer, LiCl buffer, and TE buffer), the immunoprecipitated DNA was eluted and purified for the subsequent quantitative PCR (qPCR) analysis to detect relative enrichment of each TF on the indicated genes. The PCR reaction was performed at $58^{\circ} \mathrm{C}$ for 35 cycles using $2 \times$ GoldStar Best MasterMix (Cwbiotech) with primers proximal to $\mathrm{p} 53$ or NF-KB binding sites at $\mathrm{Bax}$ and $\mathrm{BCl}-2$ promoters as follows (Bax promoter Forward: 5'-GGGTTATCTCTTGGGCTCACAA-3', Bax promoter Reverse: 5'-GAGCTCTCCCCAGCGCA -3', BCl-2 promoter Forward: 5'-TACCCAGCCTCCGTTATCCT-3', BCl-2 promoter Reverse: 5'CTTTGAGTTCGGTGGGGTCA $\left.-3^{\prime}\right)$. The PCR product was visualized by $1 \%$ agarose gel electrophoresis. Enrichment in a specific site relative to appropriate input controls is shown in the bar graph (derived from three independent experiments). The antibodies used in this assay are listed below: p53 (Abcam), NF-KBp65 (CST), Goat Anti-Mouse IgG HRP (Abcam), and Goat Anti-Rabbit IgG HRP (Abcam).

\section{RNA extraction and quantitative RT-PCR}

Total RNA was isolated with Trizol Reagent (Invitrogen) following the manufacture's protocol and was subjected to reverse transcription with GoScriptTM reverse transcription system (Promega). The coprecipitated RNA quantitative real-time RT-PCR was performed in triplicate with an Applied Biosystems Prism 7500 Fast Sequence Detection System using TaqMan universal PCR master mix according to the manufacture's protocol (Applied Biosystems Inc). Gene expression levels relative to GAPDH rRNA were calculated by a $2^{-\Delta \Delta C T}$ method. The primer sequences used in RT-PCR were shown as follows (Bax Forward: 5'-TCCACCAAGAAGCTGAGCGAG-3', Bax Reverse: 5'-GTCCAGCCCATGATGGTTCT-3', BCl-2 Forward: 5'- ATGTGTGTGGAG AGCGTCAA-3', BCl-2 Reverse: 5'-GGGCCGTACAGTTCCACAAA-3', GAPDH Forward: 5'-CGACCACTTTGTCAAGCTCA-3', GAPDH Reverse: 5'- ACTGAGTGTGGCAG

GGACTC-3').

\section{BrdU staining}

BrdU incorporation assay was carried out by following the protocol provided by Cell Signaling Technology. Briefly, BrdU was diluted to a final concentration of $0.03 \mathrm{mg} / \mathrm{mL}$ with fresh DMEM and then applied onto the cells grown on slices. Cells were incubated with $1.5 \mathrm{M} \mathrm{HCl}$ followed by 5 min fixation in $70 \%$ cold ethanol. After 3\% BSA block, Overnight immunostained with anti-BrdU antibody (CST) was then conducted as shown above. The antibody dilution ratio is 1 to 1000 . The next day, the antibodies were washed three times with PBS, stained with fluorescent secondary antibody (Abcam), and incubated at room temperature for $1 \mathrm{~h}$, and washed with PBS three times. The nucleus was visualized by DAPI staining. The representative images were captured by a Zeiss LSM510 confocal microscope (Carl Zeiss, Heidelberg, Germany)

\section{Statistical analysis}

Statistical analysis was done by using the SPSS 21 software package. Data were presented as the means \pm standard error of the means (SEM) or 
standard deviation (SD). Student $t$-test was applied to assess the statistical significance. $P$ values $<0.05$ were considered significant.

\section{DATA AVAILABILITY}

The datasets used and analyzed during the current study are available from the corresponding author on reasonable request.

\section{REFERENCES}

1. Muller PAJ, Vousden KH. p53 mutations in cancer. Nat Cell Biol. 2013;15:2-8.

2. Ghosh S, May MJ, Kopp EB. NF-kappa B and Rel proteins: evolutionarily conserved mediators of immune responses. Annu Rev Immunol. 1998;16:225-60.

3. Wu L, He Y, Hu Y, Lu H, Cao Z, Yi X, et al. Real-time surface plasmon resonance monitoring of site-specific phosphorylation of p53 protein and its interaction with MDM2 protein. Analyst 2019;144:6033-40.

4. Madan E, Parker TM, Pelham CJ, Palma AM, Peixoto ML, Nagane $M$, et al. HIFtranscribed p53 chaperones HIF-1a. Nucleic Acids Res. 2019;47:10212-34.

5. Wang $X$, Zha M, Zhao X, Jiang P, Du W, Tam AY, et al. Siva1 inhibits p53 function by acting as an ARF E3 ubiquitin ligase. Nat Commun. 2013;4:1551.

6. Prives C. Signaling to p53: breaking the MDM2-p53 circuit. Cell. 1998;95:5-8.

7. Kruiswijk F, Labuschagne CF, Vousden KH. p53 in survival, death and metabolic health: a lifeguard with a licence to kill. Nat Rev Mol Cell Biol. 2015;16:393-405.

8. Rufini A, Tucci P, Celardo I, Melino G. Senescence and aging: the critical roles of p53. Oncogene. 2013;32:5129-43.

9. Ou H-L, Schumacher B. DNA damage responses and p53 in the aging process. Blood. 2018;131:488-95.

10. Oren $M$, Rotter V. Mutant p53 gain-of-function in cancer. Cold Spring Harb Perspect Biol. 2010;2:a001107.

11. Zhang Q, Lenardo MJ, Baltimore D. 30 years of NF-KB: a blossoming of relevance to human pathobiology. Cell. 2017;168:37-57.

12. Hayden MS, Ghosh S. Shared principles in NF-kappaB signaling. Cell. 2008;132:344-62.

13. Sun S-C. The noncanonical NF-KB pathway. Immunol Rev. 2012;246:125-40.

14. Perkins ND. The diverse and complex roles of NF-KB subunits in cancer. Nat Rev Cancer. 2012;12:121-32.

15. Lwin T, Hazlehurst LA, Li Z, Dessureault S, Sotomayor E, Moscinski LC, et al. Bone marrow stromal cells prevent apoptosis of lymphoma cells by upregulation of anti-apoptotic proteins associated with activation of NF-kappaB (RelB/p52) in non-Hodgkin's lymphoma cells. Leukemia. 2007;21:1521-31.

16. Tergaonkar V, Pando M, Vafa O, Wahl G, Verma I. p53 stabilization is decreased upon NFkappaB activation: a role for NFkappaB in acquisition of resistance to chemotherapy. Cancer Cell. 2002;1:493-503.

17. Webster GA, Perkins ND. Transcriptional cross talk between NF-kappaB and p53. Mol Cell Biol. 1999;19:3485-95.

18. Ryan KM, Ernst MK, Rice NR, Vousden KH. Role of NF-kappaB in p53-mediated programmed cell death. Nature. 2000;404:892-7.

19. Zhou BP, Liao Y, Xia W, Zou Y, Spohn B, Hung MC. HER-2/neu induces p53 ubiquitination via Akt-mediated MDM2 phosphorylation. Nat Cell Biol. 2001;3:973-82.

20. Romashkova JA, Makarov SS. NF-kappaB is a target of AKT in anti-apoptotic PDGF signalling. Nature. 1999;401:86-90.

21. Rocha S, Garrett MD, Campbell KJ, Schumm K, Perkins ND. Regulation of NFkappaB and p53 through activation of ATR and Chk1 by the ARF tumour suppressor. EMBO J. 2005;24:1157-69.

22. Ito A, Lai CH, Zhao X, Saito S, Hamilton MH, Appella E, et al. p300/CBP-mediated p53 acetylation is commonly induced by p53-activating agents and inhibited by MDM2. EMBO J. 2001;20:1331-40.

23. Zhang J, Zhang Y, Xiao F, Liu Y, Wang J, Gao H, et al. The peroxisome proliferatoractivated receptor $\gamma$ agonist pioglitazone prevents NF-KB activation in cisplatin nephrotoxicity through the reduction of p65 acetylation via the AMPK-SIRT1/ p300 pathway. Biochem Pharmacol. 2016;101:100-11.

24. Tanaka T, Tsuchiya R, Hozumi Y, Nakano T, Okada M, Goto K. Reciprocal regulation of p53 and NF-KB by diacylglycerol kinase $\zeta$. Adv Biol Regul. 2016;60:15-21.

25. Huang W-C, Ju T-K, Hung M-C, Chen C-C. Phosphorylation of CBP by IKKalpha promotes cell growth by switching the binding preference of CBP from p53 to NF-kappaB. Mol Cell. 2007;26:75-87.

26. Li X, Xing D, Wang J, Zhu D-B, Zhang L, Chen X-J, et al. Effects of IkappaBalpha and its mutants on NF-kappaB and p53 signaling pathways. World J Gastroenterol. 2006;12:6658-64.

27. Bergamaschi D, Samuels $Y$, Sullivan A, Zvelebil M, Breyssens $H$, Bisso A, et al. iASPP preferentially binds p53 proline-rich region and modulates apoptotic function of codon 72-polymorphic p53. Nat Genet. 2006:38:1133-41.

28. Trigiante G, Lu X. ASPP [corrected] and cancer. Nat Rev Cancer. 2006;6:217-26.
29. Hu Y, Ge W, Wang X, Sutendra G, Zhao K, Dedeić Z, et al. Caspase cleavage of iASPP potentiates its ability to inhibit p53 and NF-KB. Oncotarget. 2015;6:42478-90.

30. Hoesel B, Schmid JA. The complexity of NF-KB signaling in inflammation and cancer. Mol Cancer. 2013;12:86.

31. Nakshatri $H$, Bhat-Nakshatri P, Martin DA, Goulet RJ, Sledge GW. Constitutive activation of NF-kappaB during progression of breast cancer to hormoneindependent growth. Mol Cell Biol. 1997;17:3629-39.

32. Bhat-Nakshatri P, Newton TR, Goulet R, Nakshatri H. NF-kappaB activation and interleukin 6 production in fibroblasts by estrogen receptor-negative breast cancer cell-derived interleukin 1alpha. Proc Natl Acad Sci USA. 1998;95:6971-6.

33. Merlin JL, Barberi-Heyob M, Bachmann N. In vitro comparative evaluation of trastuzumab (Herceptin) combined with paclitaxel (Taxol) or docetaxel (Taxotere) in HER2-expressing human breast cancer cell lines. Ann Oncol. 2002;13:1743-8.

34. Yang L, Song Z, Wang X, Yang W, Wang M, Liu H. Huaier extract enhances the treatment efficacy of paclitaxel in breast cancer cells via the NF-KB/IKBa pathway. Oncol Rep. 2017;38:3455-64.

35. Li H, Zhang W, Zhao K, Zhao D, Zheng S, Hu Y. A previously identified apoptosis inhibitor iASPP confers resistance to chemotherapeutic drugs by suppressing senescence in cancer cells. J Biol Chem. 2020;295:4049-63.

36. Kastenhuber ER, Lowe SW. Putting p53 in Context. Cell. 2017;170:1062-78.

37. Hientz K, Mohr A, Bhakta-Guha D, Efferth T. The role of p53 in cancer drug resistance and targeted chemotherapy. Oncotarget. 2017;8:8921-46.

38. Tan S-F, Dunton W, Liu X, Fox TE, Morad SAF, Desai D, et al. Acid ceramidase promotes drug resistance in acute myeloid leukemia through NF-KB-dependent P-glycoprotein upregulation. J Lipid Res. 2019;60:1078-86.

39. Ge W, Zhao K, Wang X, Li H, Yu M, He M, et al. iASPP is an antioxidative factor and drives cancer growth and drug resistance by competing with Nrf2 for Keap1 binding. Cancer Cell. 2017;32:561-73.

40. Lu M, Breyssens H, Salter V, Zhong S, Hu Y, Baer C, et al. Restoring p53 function in human melanoma cells by inhibiting MDM2 and cyclin B1/CDK1-phosphorylated nuclear iASPP. Cancer Cell. 2016:30:822-3.

41. Zhu $H$, Sun $B$, Shen Q. TNF-a induces apoptosis of human nucleus pulposus cells via activating the TRIM14/NF-KB signalling pathway. Artif Cells Nanomed Biotechnol. 2019;47:3004-12.

42. Yang JP, Hori M, Sanda T, Okamoto T. Identification of a novel inhibitor of nuclear factor-kappaB, RelA-associated inhibitor. J Biol Chem. 1999;274:15662-70.

43. Herron BJ, Rao C, Liu S, Laprade L, Richardson JA, Olivieri $E$, et al. A mutation in NFkB interacting protein 1 results in cardiomyopathy and abnormal skin development in wa3 mice. Hum Mol Genet. 2005;14:667-77.

44. Kramer D, Schön M, Bayerlová M, Bleckmann A, Schön MP, Zörnig M, et al. A proapoptotic function of iASPP by stabilizing p300 and CBP through inhibition of BRMS1 E3 ubiquitin ligase activity. Cell Death Dis. 2015;6:e1634.

\section{ACKNOWLEDGEMENTS}

We thank the staff members of Cancer Gene Function Group, School of Life Science and Technology, Harbin Institute of Technology for their discussion of the manuscript. This work was funded by the National Nature Science Foundation of China (No. 82025027, 31871389, 31301131, 31741084, and 32000517) and the Basic Science Foundation of Science and technology innovation Commission in Shenzhen (No. JCYJ20170811154452255), and China Postdoctoral Science Foundation (No. 2020M680045 and 2021T140161).

\section{AUTHOR CONTRIBUTIONS}

Y.H. conceived the project. Y.H., W.G., and X.Z wrote the paper. W.G. performed experiments and analyzed data for the most Figures in this paper. Y.W. performed experiments and analyzed data for Fig. 6C, D. S.Z, D.Z., and X.W. provided technical support for experiments.

\section{COMPETING INTERESTS}

The authors declare no competing interests.

\section{ADDITIONAL INFORMATION}

Supplementary information The online version contains supplementary material available at https://doi.org/10.1038/s41420-021-00582-1.

Correspondence and requests for materials should be addressed to X.Z. or Y.H.

Reprints and permission information is available at http://www.nature.com/ reprints 
Publisher's note Springer Nature remains neutral with regard to jurisdictional claims in published maps and institutional affiliations.

(c) Open Access This article is licensed under a Creative Commons Attribution 4.0 International License, which permits use, sharing, adaptation, distribution and reproduction in any medium or format, as long as you give appropriate credit to the original author(s) and the source, provide a link to the Creative
Commons license, and indicate if changes were made. The images or other third party material in this article are included in the article's Creative Commons license, unless indicated otherwise in a credit line to the material. If material is not included in the article's Creative Commons license and your intended use is not permitted by statutory regulation or exceeds the permitted use, you will need to obtain permission directly from the copyright holder. To view a copy of this license, visit http://creativecommons. org/licenses/by/4.0/.

(c) The Author(s) 2021 